\title{
Immunogenicity of 23-Valent Pneumococcal Polysaccharide Vaccine in Patients with Chronic Obstructive Pulmonary Disease - Hebei Province, China, September-December, 2019
}

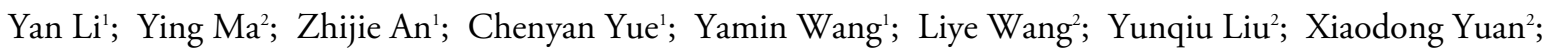 \\ Shuping Zhang'; Qiang $\mathrm{Ye}^{3}$; Hong $\mathrm{Li}^{3}$; Keli $\mathrm{Li}^{1}$; Zundong Yin'; Huaqing Wang ${ }^{1, * *}$
}

\section{Summary}

What is already known on this topic?

The global burden of chronic obstructive pulmonary disease (COPD) is serious. Pneumococcal infection is associated with acute exacerbations of COPD (AECOPD). The 23-valent pneumococcal polysaccharide vaccine (PPSV23) is recommended for COPD patients to decrease AECOPD due to pneumococcus, but evidence on the immunogenicity of PPSV23 in COPD patients is limited.

\section{What is added by this report?}

This study showed good immunogenicity of one dose of PPSV23 in COPD patients. Antibody levels against all 23 vaccine serotypes were assessed before and four weeks after vaccination of COPD patients with one dose of PPSV23. The percent of COPD patients who had two-fold increases in pneumococcal antibody levels following vaccination ranged from $65.2 \%$ (serotype 3 ) to $94.4 \%$ (serotype 2 ). There were statistically significant differences in immunogenicity by serotype. What are the implications for public health practice?

This study supports current recommendations for PPSV23 vaccination of COPD patients in China to provide protection from pneumococcal diseases.

Although 23-valent pneumococcal polysaccharide vaccine (PPSV23) is recommended for patients with chronic obstructive pulmonary disease (COPD) to prevent acute exacerbations (AECOPD) from pneumococcal diseases (1-2), the immunogenicity of PPSV23 in COPD patients is not known for all 23 vaccine serotypes. COPD prevalence and immunosenescence both increase with age, making assessment of immunogenicity important to determine. An intervention cohort study was conducted to compare antibody geometric mean concentrations (GMCs) and 2-fold increases in antibody levels before and 4 weeks after administering a dose of PPSV23 to
89 COPD patients who were invited by respiratory physicians to the study and agreed to participate. We found that PPSV23 provided good immunogenicity, with 2-fold increases in antibody levels ranging from $65.2 \%$ to $94.4 \%$, and significant increases in GMCs, but with little difference by age, presence of comorbidities, and COPD severity for most serotypes. Our findings support current recommendations to offer PPSV23 to COPD patients.

COPD represents an important public health challenge that is preventable and treatable. The disease burden of COPD is considered serious globally (1) and in China (3). Patients with COPD have frequent AECOPD that can lead to further decline in lung function, accelerating disease progression, increasing risk of death and family economic burden (4). Pneumococcus is 1 of the 3 most common bacterial antecedents to AECOPD (4). Vaccination with PPSV23 can prevent pneumococcal-related diseases.

This study was carried out in Tangshan City, Hebei Province, China between September and December 2019. Doctors from respiratory outpatient clinics of three hospitals (Majiagou, Linxi, and Kangfu) invited patients to participate in the study. Eligible patients had to have a ratio of post-bronchodilator one-second forced expiratory volume $\left(\mathrm{FEV}_{1}\right)$ to forced vital capacity (FVC) of less than 0.70 (1), be less than 80 years of age, and have stable COPD. Patients who received PPSV23 in the past 5 years or had a history of allergy to any vaccine component were excluded.

Subjects received a single intramuscular injection of $0.5 \mathrm{~mL}$ of PPSV23 that was produced by Merck Sharp \& Dohme Corporation. Venous blood samples were obtained from each subject before and 4 weeks after vaccination. Enzyme-linked immunosorbent assay (ELISA) was used to measure antibody responses for the 23 serotypes in accordance with the WHO recommendation protocol (5) by National Institute for Food and Drug Control in China. An antibody-foldincrease was calculated by dividing the post- 
vaccination antibody concentration by the prevaccination antibody concentration. GMCs and 2-fold increase rates were used as outcomes. One-half of the assay detection limit was assigned to results below the detection limit when calculating GMCs.

For the sample size calculation, the rate of 2-fold increases in antibody levels was assumed to be $70 \%$ (G) and that an acceptable 2-fold increase rate would be 50\%; a one-sided significance test was used, $\alpha=0.05$, and one-sided power $=0.80$ to determine the minimum sample size to be 73 . The sample size was targeted to 88 to allow for a $20 \%$ loss of subjects to follow-up.

EpiData (version 3.1, EpiData Software, Epi Info V6, Denmark) and SAS (version 9.4, SAS Institute, Cary, NC, USA) were used for data entry and statistical analysis. Chi-squared or Fisher's exact probability testing was used to compare 2-fold-increase rates. Student's $t$-tests or analysis of variance was used to compare GMCs. $P<0.05$ was considered a statistically significant difference.

Overall, 95 subjects were enrolled and 89 subjects completed the study; 67 subjects $(75.3 \%)$ were men; the median age was 64 years (range $47-77$ ) and $46.1 \%$ were 65 years or older; $41(46.1 \%)$ had co-morbid conditions (hypertension, diabetes, cardiovascular and cerebrovascular diseases, etc); 1 subject had received PPSV23 in the past. The distribution of COPD severity was $4(4.5 \%)$ mild, $54(60.7 \%)$ moderate, 30 $(33.7 \%)$ severe, and $1(1.1 \%)$ very severe.

The 2-fold increase rates ranged from $65.2 \%$ (serotype 3) to $94.4 \%$ (serotype 2). There were statistically significant differences in 2-fold increase rates among the 23 vaccine serotypes. Serotype 2 and 7F had the highest 2-fold increase rates $(>90 \%)$; 2-fold increase rates for serotypes $1,4,5,8,9 \mathrm{~N}, 9 \mathrm{~V}, 10 \mathrm{~A}$, $12 \mathrm{~F}, 15 \mathrm{~B}, 17 \mathrm{~F}, 18 \mathrm{C}, 19 \mathrm{~F}, 20,23 \mathrm{~F}$, and $33 \mathrm{~F}$ were between $80 \%$ and $90 \%$; 2-fold increase rates for serotype $11 \mathrm{~A}, 19 \mathrm{~A}$, and $22 \mathrm{~F}$ were between $70 \%$ and $80 \%$; and 2-fold increase rates for serotypes $3,6 \mathrm{~B}$, and 14 were less than $70 \%$. The 2 -fold increase rate for serotype $19 \mathrm{~F}$ was higher among patients with mild or moderate-severity COPD $(P=0.021)$ compared with patients with severe or very severe COPD. The 2 -fold increase rates for $8,10 \mathrm{~A}$, and $33 \mathrm{~F}$ in patients with comorbidities were lower than for patients without comorbidities $(P=0.005, P=0.016, \quad P=0.01)$. There were no other statistically significant differences by age, COPD severity, or presence of comorbidities (Table 1, Figure 1).

GMCs ranged from $0.38 \mu \mathrm{g} / \mathrm{mL}$ (serotype 3) to $6.90 \mu \mathrm{g} / \mathrm{mL}$ (serotype 14) at baseline and from
$1.03 \mu \mathrm{g} / \mathrm{mL}$ (serotype 3) to $30.36 \mu \mathrm{g} / \mathrm{mL}$ (serotype 14 ) after vaccination. There were statistically significant differences in baseline GMCs $(P<0.0001)$ and postvaccination GMCs $(P<0.0001)$ among the 23 serotypes. Compared with subjects $\geq 65$ years, younger subjects had lower baseline GMCs for serotype 9V $(P=0.035)$ and higher post-vaccination GMC for serotype $10 \mathrm{~A}(P=0.030)$. After vaccination, GMCs of serotype $6 \mathrm{~B}$ and $19 \mathrm{~F}$ among patients with mild or moderate COPD were higher than among patients with severe and very severe COPD $(P=0.04, P=0.039)$. There were no other significant differences in GMCs by age, COPD severity, or presence of comorbidities (Figure 1, Supplementary Table S1, available in http://weekly.chinacdc.cn/).

\section{DISCUSSION}

This study found that immunogenicity provided by 1 dose of PPSV23 in COPD patients was good. Although immunogenicity varied by serotype, it varied little by age, presence of comorbidities, and severity of COPD for most of the vaccine serotypes. This is the first study to assess immunogenicity of all 23 serotypes from one dose of PPSV23 in COPD patients. The findings support current recommendations to offer PPSV23 to COPD patients in China to provide protection from pneumococcal disease and prevent or reduce the number of AECOPD.

There have been some evaluations of PPSV23 immunogenicity in COPD patients. A study conducted in Taiwan, China assessed the immunogenicity of 8 PPSV23 serotypes (4, 6B, 7F, $9 \mathrm{~V}, 14,18 \mathrm{C}, 19 \mathrm{~F}$, and $23 \mathrm{~F}$ ) in COPD patients (6). Two-fold increases for serotypes $6 \mathrm{~B}$ and 14 and postvaccination GMCs of the 8 corresponding serotypes in this study were lower than that in the study conducted in Taiwan, China. Differences between the two studies may be due to different blood collection intervals (4 weeks vs. 6 weeks) or different ELISA methods.

Two studies conducted in Japan among COPD patients showed lower 2-fold increases and postvaccination GMCs for $6 \mathrm{~B}, 14,19 \mathrm{~F}$, and $23 \mathrm{~F}$ than in this study (7-8). Differences between the studies may have been due to different pre-vaccination antibody concentrations or different characteristics of subjects. In this study, pre-vaccination GMCs for serotype 6B, $19 \mathrm{~F}$, and $23 \mathrm{~F}$ were lower than that in the study conducted in Japan ( 7 ). The median age in this study was younger and fewer comorbid illnesses were considered to be other respiratory diseases than that in 
TABLE 1. Two-fold increase rates (\%) of 23 serotypes and 95\% confidence intervals after PPSV23 vaccination in COPD patients in Tangshan City, Hebei Province, China during September to December 2019.

\begin{tabular}{|c|c|c|c|c|c|c|c|}
\hline \multirow[b]{2}{*}{ Serotype } & \multicolumn{2}{|c|}{ Age group } & \multicolumn{2}{|c|}{ COPD severity } & \multicolumn{2}{|c|}{ Comorbidities } & \multirow[b]{2}{*}{ Total } \\
\hline & $<65$ years & $\geq 65$ years & $\begin{array}{l}\text { Mild and } \\
\text { moderate }\end{array}$ & $\begin{array}{c}\text { Severe and } \\
\text { very severe }\end{array}$ & Yes & No & \\
\hline 1 & $89.6(77.3-96.5)$ & $82.9(67.9-92.9)$ & $87.9(76.7-95.0)$ & $83.9(66.3-94.6)$ & $80.5(65.1-91.2)$ & $91.7(80.0-97.7)$ & $86.5(77$ \\
\hline 2 & $95.8(85.8-99.5)$ & 92.7 (80.1-98.5) & $96.6(88.1-99.6)$ & $90.3(74.3-98.0)$ & $92.7(80.1-98.5)$ & 35.8-99.5) & $94.4(87.4$ \\
\hline 3 & $68.8(53.8-81.3)$ & $61.0(44.5-75.8)$ & $62.1(48.4-74.5)$ & $71.0(52.0-85.8)$ & $63.4(46.9-77.9)$ & $66.7(51.6-79.6)$ & $65.2(54.3-75.0)$ \\
\hline 4 & $85.4(72.2-93.9)$ & $82.9(67.9-92.9)$ & $84.5(72.6-92.7)$ & $83.9(66.3-94.6)$ & $78.1(62.4-89.4)$ & $89.6(77.3-96.5)$ & $84.3(75.0-91.1)$ \\
\hline 5 & $85.4(72.2-93.9)$ & $85.4(70.8-94.4)$ & $86.2(74.6-93.9)$ & $83.9(66.3-94.6)$ & $80.5(65.1-91.2)$ & $89.6(77.3-96.5)$ & $85.4(76.3-92.0)$ \\
\hline $6 \mathrm{~B}$ & $66.7(51$ & 68.3 & $72.4(59$ & 58. & 68.3 & 66 & 67. \\
\hline $7 \mathrm{~F}$ & $93.8(82.8-98.7)$ & 87.8 & $91.4(81.0-97.1)$ & $90.3(74.3-98.0)$ & $87.8(73.8-95$ & 93. & 91 \\
\hline 8 & $89.6(77.3-96.5)$ & $87.8(73$ & $87.9(76.7-95.0)$ & $90.3(74.3-98.0)$ & $78.1(62.4-89.4)$ & 97.9 (88.9-99.9) & $88.8(80.3-94.5)$ \\
\hline $9 \mathrm{~N}$ & $95.8(85.8-\varsigma$ & $82.9(67$. & $89.7(78.8-96.1)$ & $90.3(74.3-98.0)$ & $85.4(70.8-94.4)$ & $93.8(82.8-98.7)$ & $89.9(81.7-$ \\
\hline $9 \mathrm{~V}$ & $89.6(77.3-96.5)$ & $85.4(70.8$ & $89.7(78.8-96.1)$ & $83.9(66.3-94.6)$ & $85.4(70.8-94.4)$ & $89.6(77.3-96.5)$ & $87.6(79.0-93.7)$ \\
\hline $10 \mathrm{~A}$ & $91.7(80.0-97.7)$ & $78.1(62.4$ & $86.2(74.6-93.9)$ & $83.9(66.3-94.6)$ & $75.6(59.7-87.6)$ & $93.8(82.8-98.7)$ & $85.4(76.3-92.0)$ \\
\hline $11 \mathrm{~A}$ & $77.1(62.7-88.0)$ & $70.7(54.5$ & $74.1(61.0-84.7)$ & $74.2(55.4-88.1)$ & $75.6(59.7-87.6)$ & $72.9(58.2-84.7)$ & $74.2(63.8-82.9)$ \\
\hline $12 \mathrm{~F}$ & $91.7(80.0-97.7)$ & 87.8 (73.8-95.9) & $93.1(83.3-98.1)$ & $83.9(66.3-94.6)$ & $87.8(73.8-95.9)$ & $91.7(80.0-97.7)$ & $89.9(81.7-95.3)$ \\
\hline 14 & $70.8(55.9-83.1)$ & $65.9(49.4-79.9)$ & $72.4(59.1-83.3)$ & $61.3(42.2-78.2)$ & $75.6(59.7-87.6)$ & $62.5(47.4-76.1)$ & $68.5(57.8-78.0)$ \\
\hline $15 B$ & $85.4(72.2-93.9)$ & $90.2(76.9-97.3)$ & $87.9(76.7-95.0)$ & $87.1(70.2-96.4)$ & $82.9(67.9-92.9)$ & $91.7(80.0-97.7)$ & 87.6 \\
\hline $17 \mathrm{~F}$ & $89.6(77.3-96.5)$ & 87.8 & $91.4(81.0-97.1)$ & 83.9 & $85.4(70.8-94.4)$ & $91.7(80$ & $88.8(8$ \\
\hline $18 \mathrm{C}$ & $93.8(82.8-98.7)$ & 80.5 & $89.7(78.8-96.1)$ & $83.9(66.3-94.6)$ & $82.9(67.9-92.9)$ & $91.7(80.0-97.7)$ & $87.6(79.0-9$ \\
\hline $19 A$ & $79.2(65.0-89.5)$ & 75.6 (59.7-87.6) & $79.3(66.7-88.8)$ & $74.2(55.4-88.1)$ & $73.2(57.1-85.8)$ & $81.3(67.4-91.1)$ & $77.5(67.5-85.7)$ \\
\hline $19 \mathrm{~F}$ & $83.3(69.8-92.5)$ & $78.1(62.4-89.4)$ & $87.9(76.7-95.0)$ & $67.7(48.6-83.3)$ & $75.6(59.7-87.6)$ & $85.4(72.2-93.9)$ & $80.9(71.2-88.5)$ \\
\hline 20 & $83.3(69.8-92.5)$ & $80.5(65.1-91.2)$ & $82.8(70.6-91.4)$ & $80.7(62.5-92.6)$ & $80.5(65.1-91.2)$ & $83.3(69.8-92.5)$ & $82.0(72.5-8$ \\
\hline $22 \mathrm{~F}$ & $2.7-88.0)$ & $68.3(51$. & $77.6(64.7-87.5)$ & $64.5(45.4-80.8)$ & $68.3(51.9-81.9)$ & $77.1(62.7$ & $73.0(62$ \\
\hline $23 \mathrm{~F}$ & $85.4(72.2-93.9)$ & 80.5 (65.1-91.2) & $82.8(70.6-91.4)$ & $83.9(66.3-$ & $82.9(67.9-92.9)$ & $83.3(69.8-9$ & $83.2(73.7-90.3)$ \\
\hline $33 \mathrm{~F}$ & $93.8(82.8-98.7)$ & $85.4(70.8-94.4)$ & $91.4(81.0-97.1)$ & $87.1(70.2-96.4)$ & $80.5(65.1-91.2)$ & $97.9(88.9-99.9)$ & $89.9(81.7-95.3)$ \\
\hline
\end{tabular}

Abbreviations: PPSV23=23-valent pneumococcal polysaccharide vaccine; COPD=chronic obstructive pulmonary disease.

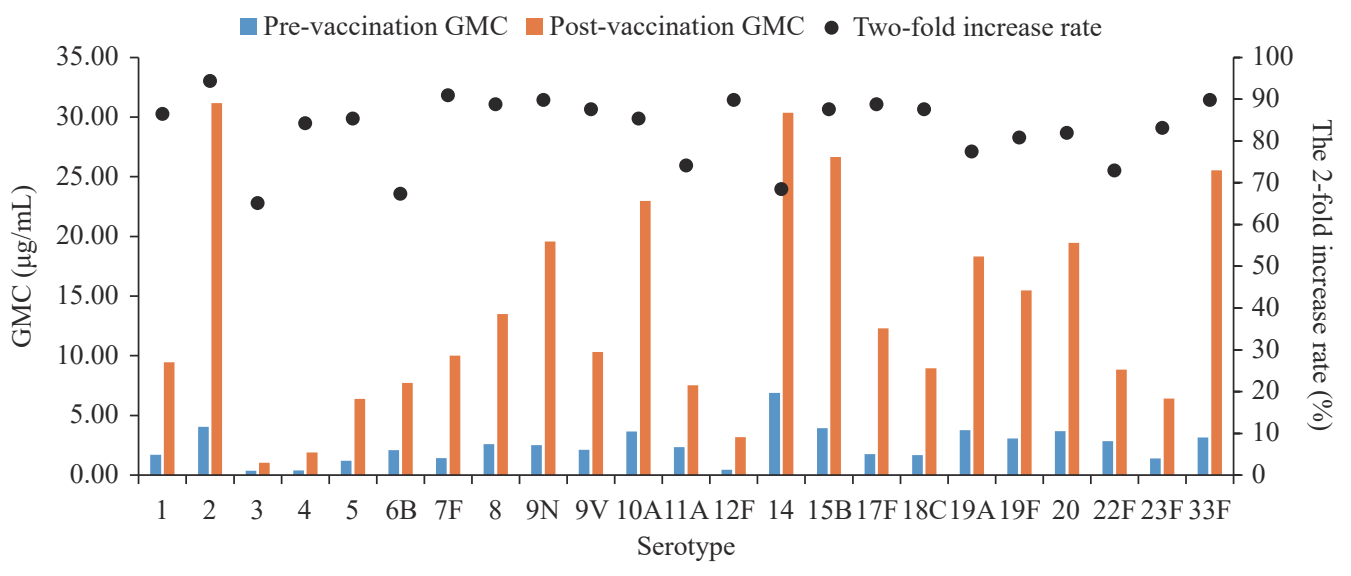

FIGURE 1. Geometric mean concentrations (GMCs) pre- and post-vaccination and 2-fold increase rates of 23 serotypes post 23-valent pneumococcal polysaccharide vaccination in patients with chronic obstructive pulmonary disease in Tangshan City, Hebei Province, China during September to December 2019. 
the study conducted in Japan (8).

The immune response induced by PPSV23 in COPD patients appears to be comparable with those of age-matched healthy adults. One study assessed immunogenicity of PPSV23 in COPD patients and healthy controls and found that there were no statistically significant differences in the antibody GMCs between COPD patients and healthy controls (9). The 2-fold increase rates of 23 serotypes in this study were not lower than those in the Phase III licensure clinical trial of PPSV23 conducted in China (10).

This study was subject to at least four limitations. First, there were no healthy controls to assess differences in immune response between COPD patients and healthy adults. Other studies have shown comparable immunogenicity, however. Second, subjects were not selected randomly, which could affect representativeness. Third, the sample size was too small to assess immunogenicity among subgroups. Finally, the study was short term and did not provide evidence of persistence of immunity. There are several studies reporting 1-2 years of immune persistence following PPSV23 vaccination of COPD patients $(6-7,9)$. Only one study reported long-term persistence (8). Following these subjects for an additional 4-5 years could provide additional evidence of persistence.

Antibody levels that correlate with protection from pneumococcal disease have not been clearly defined for adults. Thus, the relationship between immunogenicity and effectiveness is unclear. This important relationship merits additional research using experimental study designs to determine health and economic benefits of PPSV23 in COPD patients.

In conclusion, the immunogenicity of one dose of PPSV23 is good in COPD patients. PPSV23 vaccination should continue to be recommended for COPD patients in China.

Acknowledgments: Jianquan Li, Xiaohui Zhang, Yi Wang, Yanru Zhang, Yadi Su, Xueying Li, and other colleagues; Lance Rodewald, MD.

Conflicts of interest: We declare no competing interests. The funders had no role in the design of the study; in the collection, analyses, or interpretation of data; in the writing of the manuscript, or in the decision to publish the results.

Funding: National Key R\&D Program of China

\section{(2017YFC1309304).}

doi: $10.46234 / \mathrm{ccdcw} 2021.089$

\# Corresponding author: Huaqing Wang, wanghq@chinacdc.cn.

${ }^{1}$ National Immunization Program, Chinese Center for Disease Control and Prevention, Beijing, China; ${ }^{2}$ Kailuan General Hospital, Tangshan, Hebei, China; ${ }^{3}$ National Institute for Food and Drug Control, Beijing, China.

Submitted: March 15, 2021; Accepted: April 08, 2021

\section{REFERENCES}

1. Vogelmeier CF, Criner GJ, Martinez FJ, Anzueto A, Barnes PJ, Bourbeau J, et al. Global Strategy for the Diagnosis, Management, and Prevention of Chronic Obstructive Lung Disease 2017 Report: GOLD Executive Summary. Eur Respir J. 2017;49(3):1700214. doi: 10.1183/13993003.00214-2017.

2. ACIP. Prevention of Pneumococcal Disease - Recommendations of the Advisory Committee on Immunization Practices (ACIP). Morbidity and Mortality Weekly Report [recommendations and reports series], 1997, 46:RR-8. https://www.cdc.gov/mmwr/pdf/rr/rr4608.pdf.

3. Fang LW, Gao P, Bao HL, Tang X, Wang BH, Feng YJ, et al. Chronic obstructive pulmonary disease in China: a nationwide prevalence study. Lancet Respir Med 2018;6(6):421 - 30. http://dx.doi.org/10.1016/ S2213-2600(18)30103-6.

4. Expert Group on Diagnosis and Treatment of Acute Exacerbation of Chronic Obstructive Pulmonary Disease. Chinese expert consensus on diagnosis and treatment of acute exacerbation of chronic obstructive pulmonary disease (AECOPD)(2017 update). Int Respir J 2017; 37(14):1041 - 57. http://dx.doi.org/10.3760/cma.j.issn.1673-436X. 2017.14.001. (In Chinese).

5. World Health Organization. Training manual for Enzyme linked immunosorbent assay for the quantitation of Streptococcus pneumoniae serotype specific IgG (Pn PS ELISA) (007sp Version). https://www. vaccine.uab.edu/uploads/mdocs/ELISAProtocol(007sp).pdf. [2021-225].

6. Lai CC, Lee LN, Yu CJ, Hsueh PR, Yang PC, Kuo SH, et al. Antibody responses to pneumococcal polysaccharide vaccine in Taiwanese patients with chronic obstructive pulmonary disease. J Formos Med Assoc 2007;106(3):196 - 203. http://dx.doi.org/10.1016/S0929-6646 (09)60240-0.

7. Chen M, Hisatomi Y, Furumoto A, Kawakami K, Masaki H, Nagatake $\mathrm{T}$, et al. Comparative immune responses of patients with chronic pulmonary diseases during the 2-year period after pneumococcal vaccination. Clin Vaccine Immunol 2007;14(2):139 - 45. http://dx. doi.org/10.1128/CVI.00336-06.

8. Ohshima N, Nagai H, Matsui H, Akashi S, Makino T, Akeda Y, et al. Sustained functional serotype-specific antibody after primary and secondary vaccinations with a pneumococcal polysaccharide vaccine in elderly patients with chronic lung disease. Vaccine 2014;32(10):1181 6. http://dx.doi.org/10.1016/j.vaccine.2013.09.060.

9. de Roux A, Schmidt N, Rose M, Zielen S, Pletz M, Lode H. Immunogenity of the pneumococcal polysaccharide vaccine in COPD patients. The effect of systemic steroids. Respir Med 2004;98(12): 1187 - 94. http://dx.doi.org/10.1016/j.rmed.2004.04.012.

10. Huang LL, Wang L, Li H, Hu YS, Ru WP, Han WX, et al. A phase III clinical trial to evaluate the safety and immunogenicity of 23-valent pneumococcal polysaccharide vaccine (PPV23) in healthy children, adults, and elderly. Hum Vaccin Immunother 2019;15(1):249 - 55. http://dx.doi.org/10.1080/21645515.2018.1509648. 


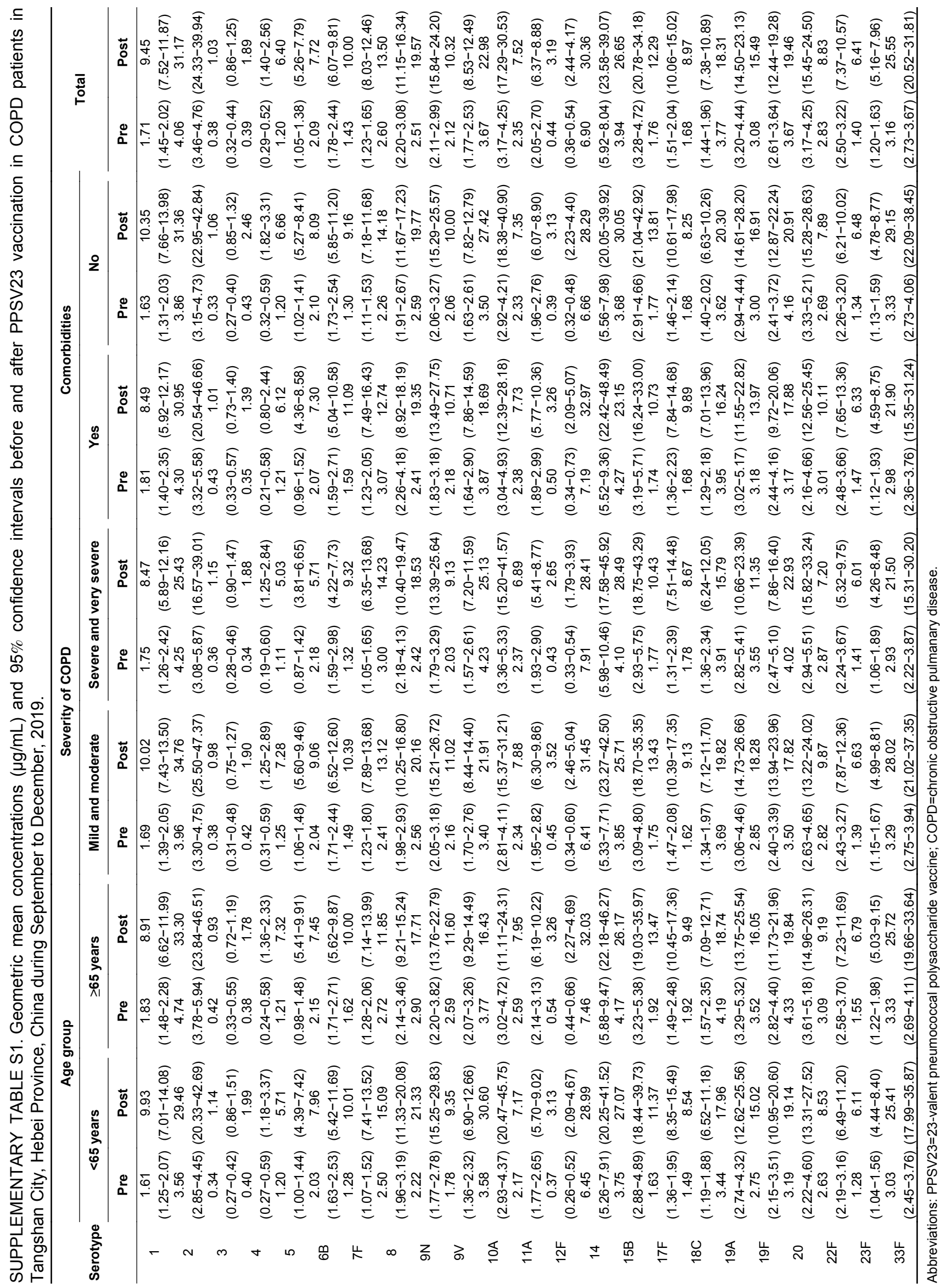

